\title{
le portiQue $\quad$ Portique
}

Revue de philosophie et de sciences humaines

5 | 2000

Passages du siècle

Le $\mathrm{xx}^{\mathrm{e}}$ siècle, un événement, un objet. La Modernité, époque byzantine de la culture?

\section{Yvan Elissalde}

\section{OpenEdition \\ Journals}

Édition électronique

URL : http://journals.openedition.org/leportique/404

DOI : $10.4000 /$ leportique.404

ISSN : $1777-5280$

Éditeur

Association "Les Amis du Portique"

Édition imprimée

Date de publication : 1 janvier 2000

ISSN : 1283-8594

Référence électronique

Yvan Elissalde, «Le $x x^{e}$ siècle, un événement, un objet. La Modernité, époque byzantine de la culture? », Le Portique [En ligne], 5 | 2000, mis en ligne le 24 mars 2005, consulté le 25 mars 2021. URL : http://journals.openedition.org/leportique/404; DOI : https://doi.org/10.4000/leportique.404

Ce document a été généré automatiquement le 25 mars 2021.

Tous droits réservés 


\title{
Le $\mathrm{xx}^{\mathrm{e}}$ siècle, un événement, un objet. La Modernité, époque byzantine de la culture?
}

\author{
Yvan Elissalde
}

Introduction

1 Deux préalables à la mise en place de la discussion s'avèrent nécessaires : d'une part, définir d'un peu près le byzantinisme; d'autre part relier le thème du byzantinisme et les concepts d'interprétation et de Modernité. Ces éclaircissements ayant été donnés, nous nous attacherons à mettre en doute cette corrélation; enfin, nous essaierons de passer outre ces difficultés et de montrer quels gains spéculatifs non négligeables on est en droit d'espérer de la sauvegarde d'une notion qui à première vue, et compte tenu des apports des diverses philosophies herméneutiques $\mathrm{du} \mathrm{xx}^{\mathrm{e}}$ siècle, paraît peu défendable.

2 Il n'est pas évident, en effet, de poser sans autre procès que le byzantinisme est par soi un phénomène de type herméneutique, si, par byzantinisme, on entend en général « la tendance aux discussions qui évoquent, par leur excès de subtilité, leur caractère formel et oiseux, les disputes théologiques de la Byzance du Bas-Empire et du Moyen Âge ». Cette définition usuelle ne montre pas de lien analytique entre le byzantinisme et les pratiques interprétatives, étant donné que toute discussion excessivement subtile, formelle, oiseuse et de type théologique, n'est pas intrinsèquement ni nécessairement herméneutique.

3 Partons donc, pour situer la discussion dans le champ proprement herméneutique, de la définition que G. Steiner donne du byzantinisme dans Le Sens $d u$ sens ${ }^{1}$ :

"Les périodes, les climats culturels où dominent le critique et l'exégète sont qualifiés d'“Alexandrins" ou de "Byzantins". Cet épithète fait référence à la primauté des techniques et d'idéaux relatifs à la grammatologie, à l'édition, à la didactique, à l'établissement de glossaires et au jugement sur toute créativité poético-esthétique réelle, caractéristique de l'Alexandrie hellénistique et de la Byzance du Bas-Empire et du Moyen Âge. Elles sont révélatrices de l'impérialisme du commentaire et du commentaire sur le commentaire. À notre époque, le nom 
d'une seule métropole ne suffit pas à désigner un phénomène analogue à Alexandrie ou à Byzance. Peut-être notre époque sera-t-elle connue comme celle des annotateurs, des clercs qui exercent sur la place publique ».

4 Commentons rapidement, pour les faire ressortir, les éléments significatifs de cette citation. La définition de Steiner a le mérite de relier directement le byzantinisme et les pratiques interprétatives. Il y précise d'une part que le byzantinisme est par soi un phénomène d'excès interprétatif, et d'autre part que cet excès est analysable en termes de pouvoir (" primauté », « domination », « impérialisme »), ce qui suppose un rapport entre l'agent du pouvoir (désigné comme étant le commentaire ainsi que son producteur, l'herméneute en général) et son patient (représenté ici par la créativité). Par ailleurs, l'impérialisme de l'interprétation qu'est le byzantinisme se joue, selon Steiner, sur le double plan de la technique (il est question de l'herméneutique en son sens classique de "technologie de la compréhension », pour reprendre une expression employée parfois par Gadamer) et de l'idéal. L'excès n'est donc pas seulement dans l'activité (un excès quantitatif, c'est-à-dire une disproportion numéraire entre les œuvres de création et les œuvres d'interprétation) mais aussi dans la valeur (un excès beaucoup plus qualitatif, consistant à attribuer trop de valeur à l'activité interprétative au détriment de l'activité créatrice). Enfin, Steiner se permet de caractériser notre époque par le byzantinisme ainsi défini, c'est-à-dire de définir la Modernité culturelle comme l'époque où le pouvoir de l'interprétation excéderait en quantité, par sa production, et en qualité, par ses valeurs, le pouvoir de la création.

I. Problèmes du byzantinisme

5 Une telle présentation des choses est problématique à plus d'un titre, et nous allons nous attacher à mettre à la question sa validité. Premièrement, à supposer que la Modernité se caractérise par l'excès byzantin d'interprétation, cette caractérisation ne lui est pas propre, puisqu'elle peut s'appliquer à d'autres époques de la culture. L'analyse de la Modernité en termes de byzantinisme risque donc de rater ce qui fait sa spécificité. Penser la Modernité par une notion qui chapeaute aussi bien la culture alexandrine que byzantine, et qui pourrait encore s'appliquer, si l'on en croit par exemple Montaigne, à la Renaissance, n'est-ce pas la penser en renonçant à la saisir de l'intérieur, par ses qualités intrinsèques? Telle est la première difficulté opposable à l'idée que la Modernité est l'époque byzantine de la culture.

6 Par ailleurs, cette appréciation ne se rend-elle pas coupable de ce qu'on pourrait appeler l'oubli de l'histoire? Car le byzantinisme se réfère sans complexes historiographiques à la Byzance du Bas-Empire et du Moyen Âge, comme si on pouvait transposer la situation de cette culture à notre situation culturelle. Mais, outre que la charge péjorative du mot «byzantinisme » risque d'être une injustice faite à la réalité culturelle de cette époque, qui est loin d'être homogène ${ }^{2}$, comment peut-on mettre dans un même sac deux époques aussi étrangères, sans nier l'efficace du temps ainsi que l'historicité de l'interprétation elle-même en ses pratiques et en ses concepts? Faire comme si ceux-ci ne présentaient pas des configurations sui generis?

7 La troisième difficulté qui attend celui qui voudrait employer la notion de byzantinisme pour caractériser la Modernité peut se formuler dans la question suivante : cette notion n'est-elle pas dangereusement anti-intellectualiste? On peut penser qu'elle risque en particulier de faire le jeu de l'opinion qui évalue négativement les pratiques interprétatives que sont le commentaire érudit, la philologie, la critique esthétique, littéraire, ou encore l'exégèse. Le vulgaire n'est-il pas toujours prompt à déconsidérer 
l'interprétation, comme recherche prétendument vouée à l'échec parce qu'interminable? Le thème populaire des "gloses sans fin" fustige l'activité interprétante, soit que son indéfinité même la disqualifierait, soit qu'elle se ridiculiserait en portant sur des points insignifiants comme «le sexe des anges", thème qui fut, comme on sait, âprement débattu par les théologiens byzantins. L'appréciation de byzantinisme est donc à elle seule, par la dose de mépris qui lui est intrinsèque, une charge contre l'intellectualisme herméneutique en général, objet d'une dévaluation commune qui, incapable de voir dans l'interprétation sans fin la structure et richesse d'un sens inépuisable, commet un contresens et prend l'inextinguibilité herméneutique pour l'incontinence d'un bavardage de haute volée, cela dit pour rester poli. Cela ne suffirait-il pas à tout intellectuel, à tout amoureux du langage, de l'histoire et de la compréhension, à tout philosophe, pour prendre ses distances avec une telle appréciation?

8 Ce n'est pas tout. La seconde série d'apories, de loin les plus fécondes, concerne les présupposés théoriques de la définition du byzantinisme. L'un d'entre eux consiste dans le thème de l'excès quantitatif et qualitatif de l'interprétation. Mais l'interprétation n'est-elle pas par essence en excès par rapport à son objet intentionnel ? La disproportion dénoncée par Steiner entre interprétation et création ne paraît pas pouvoir caractériser une époque culturelle, mais bien faire partie de l'interprétation en propre, toutes époques confondues, si l'on s'avise de ce que l'interprétation est par soi plurielle et la création singulière. Il en va ici du concept d'interprétation comme de celui d'hypothèse : on ne peut parler d'interprétation que lorsqu'on a pris conscience d'une multiplicité d'interprétations réelles ou simplement possibles, si bien qu'une interprétation ne se connaît comme telle que par rapport aux autres interprétations gravitant autour de la création singulière. Par suite, être surnuméraire n'est pas un accident de l'interprétation, mais un trait constitutif qui régit le rapport proprement herméneutique entre œuvre et commentaire de l'œuvre. Celui-ci n'est pas caractérisable comme le rapport pauvrement binaire entre la singularité de la création et la singularité d'une interprétation, mais comme le rapport multiple entre la création et les interprétations qui multiplient, développent, manifestent, disséminent le sens de l'œuvre. Si l'aspect surnuméraire est inscrit au cœur de l'interprétation comme telle, il est impossible d'y voir un aspect contingent, appartenant à certaines époques en propre, et impossible de le dénoncer comme excessif, puisqu'il ne peut en être autrement. On ne reproche pas à un pommier de produire des pommes.

9 S'ensuit une autre difficulté, sans doute la plus considérable et la plus intéressante, concernant encore plus directement la notion d'excès. Celle-ci n'a de sens, en effet, qu'en référence à une limite transgressée ou transgressable. Il faut donc être en mesure d'assigner une ou des limites à l'interprétation, pour pouvoir parler d'excès interprétatif et donc de byzantinisme. Quelles sont ses limites ? Il n'est pas simple de le dire, comme l'a éprouvé l'auteur de L'Euvre ouverte, qui, dans son souci tardif mais bienvenu de ne pas ouvrir démesurément l'œuvre, et de la fermer à la surinterprétation déconstructionniste ou hermétique ${ }^{3}$ a bien du mal à défendre les droits de l'œuvre (intentio operis) contre ceux du lecteur (intentio lectoris). Pour Steiner, il semble que ce soit la création elle-même, à titre de patient du pouvoir de l'interprétation, qui fonctionne comme limite de l'interprétation, en ce que l'interprétation est dite excessive quand elle prime, domine, conquiert en quantité et en qualité le champ culturel en droit occupé par les créations. Le présupposé aussi massif que fragile n'est-il 
pas, ici, le dualisme entre l'œuvre et l'interprétation? Pour que la création limite l'interprétation, encore faudrait-il prouver que la création est distincte de ses interprétations, et l'auteur opposable à ses interprètes. Or, un des principaux apports de la Modernité culturelle en général, repris et amplifié par les philosophies herméneutiques $d u x^{e}$ siècle en particulier, consiste précisément à contester ce dualisme, en lui substituant un rapport ontologique ou critique de constitution réciproque. Qu'on pense par exemple aux concepts gadameriens de jeu ou d'application: ils ont pour sens de montrer que l'interprétation n'est pas un ajout contingent scotché à l'œuvre et lui restant extérieur, mais bien plutôt un prolongement et un achèvement de l'œuvre par la manifestation pratique de son sens, par la configuration et l'apparaître phénoménologique de ce sens. De même, l'interprète et l'auteur ne sont pas des personnages isolables l'un de l'autre, puisque l'un comme l'autre et l'un par l'autre, à travers l'épaisseur scripturaire de leurs œuvres propres, travaillent à laisser se dire et venir en présence la chose même, un être, une vérité historialement constituée qu'ils visent également. Une autre manière, toujours phénoménologique, de corroder la distinction auteur/interprète consiste à étendre le concept d'interprétation en amont à l'activité de l'auteur et en aval à celle du spectateur, si bien que F. Fédier ${ }^{4}$, entre autres, n'hésite pas à nier la différence entre créateur, interprète et public en affirmant que «tous les êtres humains pratiquent l'interprétation ", c'est-à-dire accueillent, configurent, donnent forme au phénomène du monde ; interprétation dite " du premier rang » pour le créateur, " du second rang " pour l'interprète proprement dit et "du troisième rang" pour le public. Mais la conception phénoménologique de l'interprétation n'est pas la seule à saper l'opposition éculée entre auteur et interprète, œuvre et interprétation : la critique littéraire, de Du Bos à J. Derrida, en passant par R. Barthes, la dénonce depuis longtemps, en montrant cette fois, non que le créateur est un interprète, mais que l'interprète est un créateur. Du Bos homogénéise leur activité en déclarant que « la voie de la critique idéale [...] est la voie de la production, mais parcourue en sens inverse, le critique ayant pour point de départ le point d'arrivée du créateur et pour point d'arrivée son point de départ ${ }^{5}$ ", ce qui fait de l'interprète un créateur au second degré, dans la mesure ou sa création a pour matière première la création d'un autre. R. Barthes affirme lui que "Autrefois séparés par le mythe usé du superbe créateur et de l'humble serviteur, tous deux nécessaires, chacun à leur place, etc., l'écrivain et le critique se rejoignent dans la même condition difficile, face au même objet : le langage ${ }^{6} »$. J. Derrida, quant à lui, en récusant que l'intention de l'auteur puisse assurer la présence stable et close d'un sens dans les signes du texte, ouvre à l'infini l'œuvre à l'intention du lecteur promu au rang de créateur non pas secondaire ou dérivé, mais autonome et de plein droit, égal en dignité créatrice à l'auteur. Toutes ces manières de nier la scission ou l'indépendance conceptuelle et pratique entre création et interprétation sont il est vrai très diverses, mais il est clair qu'elles convergent toutes vers les avancées contemporaines de l'analyse du langage qui forment leur fondement et assure leur légitimité. C'est aussi en fonction de la place centrale accordée au langage que, pour penser l'interprétation, il est convenu, de nos jours, d'enraciner les analyses dans le Peri Hermèneias d'Aristote, référence qui permet de corréler parole et interprétation, parce qu'elle permettrait de montrer non seulement que l'interprétation est langage, mais encore et surtout que le langage en général est par soi interprétatif, est un phénomène herméneutique de part en part, que ce langage soit celui de l'interprète stricto sensu ou celui de l'auteur d'une œuvre. Ce qui importe à ceux qui suivent les leçons du Stagirite, c'est de souligner que 
la seule différence entre parler ou écrire et interpréter, est une différence somme toute mineure de sens, au sens d'orientation, c'est-à-dire que l'interprétation serait le parcours en sens inverse de l'expression. J. Pépin ${ }^{7}$, par exemple, note que l'expression, opération ad extra, va de la pensée aux signes et l'interprétation, opération ad intra, des signes à la pensée. Mais le chemin herméneutique qui monte et qui descend n'est-il pas le même ? Ce chemin commun, ce médium de l'expression et de l'interprétation, et, par suite, de la création et du commentaire, qu'est-ce, sinon le langage dans sa quadruple modalité : parole aussi bien qu'écoute, écriture aussi bien que lecture?

Dans ces conditions, ne peut-on pas douter radicalement de la pertinence de la notion de byzantinisme? Celle-ci semble reposer sur des présupposés inacceptables concernant la notion d'interprétation; sur un anti-intellectualisme aussi injuste que primaire; sur des dualismes désormais caduques (création/interprétation; auteur/ interprète) tant $\mathrm{du}$ point de vue des théories herméneutiques que des pratiques interprétatives; sur des oublis aussi massifs que l'oubli de l'histoire et l'oubli du langage.

II. Apologie du byzantinisme

11 Quel intérêt y aurait-il donc à sauver de la caducité la notion de byzantinisme, dans le cadre d'une pensée de l'interprétation et de la Modernité ? Et comment lui faire surmonter toutes les apories repérées? Plusieurs arguments nous encourageraient à ne pas nous en débarrasser trop vite. Pour les saisir, il convient de raisonner par l'absurde, c'est-à-dire d'envisager quelles conséquences également inacceptables sont déductibles de la négation de tout byzantinisme, défini comme l'excès interprétatif. On verra alors en quel sens on peut parler de la Modernité en termes de byzantinisme, quitte à apporter quelques retouches à la définition de G. Steiner.

Nier l'effectivité et la possibilité d'un excès interprétatif, c'est poser que l'interprétation est infinie en droit et indéfinie en fait. On peut se réjouir phénoménologiquement de cette infinité, en la comprenant dans le thème positif de l'inépuisabilité des possibilités de sens d'une œuvre, c'est-à-dire de l'illimitation nécessaire des manifestations et des configurations de l'apparaître de ce sens. Il n'y a de sens que compris, et de compréhension que dans une reprise interprétative sans fin de cette compréhension explicitée dans l'élément du langage, c'est-à-dire interprétée. Paradoxalement, l'illimitation de l'interprétation est fondée sur la limite du sujet de l'interprétation : c'est la finitude de la conscience, conçue comme instance réceptrice du sens, qui rend nécessaire ce que P. Ricœur appelle un «Cogito médiatisé par toute l'épaisseur des signes ", c'est-à-dire un Cogito qui s'auto-constitue herméneutiquement par la médiation sans fin de la compréhension des signes d'autrui. Mais le risque, ici, n'est-il pas d'omettre de fournir les moyens conceptuels permettant de faire la part entre un bon et un mauvais infini? L'absence de limites induite par la négation de tout byzantinisme permet, certes, de penser "soi-même comme un autre ", mais en même temps, ne donne-t-elle pas une licence dangereuse à la surinterprétation et à la mésinterprétation, c'est-à-dire à l'exercice intempérant d'un type d'interprétation qui se croit tout permis, sous prétexte nietzschéen (certes mal interprété lui-même) qu'« il n'y a pas de faits, mais seulement une interprétation des faits »; pas même de textes, mais seulement une interprétation des textes? Pour reprendre le bon mot de A. Todorov cité par U. Eco ${ }^{8}$, peut-on accepter qu'un texte ne soit qu'« un pique-nique où l'auteur apporte les mots et le lecteur le sens »? L'illimitation de l'interprétation peut donc présenter pour premier inconvénient théorique de ne pas assurer la défense 
de l'art d'interpréter, c'est-à-dire le suivi rigoureux et difficile de règles herméneutiques, contre la fantaisie interprétative la plus débridée. Comment faire la part entre les bonnes interprétations et les mauvaises? Les raisonnables et les délirantes? Entre interprétations et surinterprétations? Entre les interprétations fidèles, quoique plurielles, et les libres interprétations, aussi bien celle qui se reconnaît pour telle en assumant ouvertement les libertés qu'elle prend avec le texte, que celle qui fait violence au texte en prétendant en découvrir les arcanes ou la richesse sans fond?

13 Malgré tout le respect que on peut avoir pour la question byzantine du sexes des anges, dont on s'est, il est vrai, un peu facilement moqué, force est de reconnaitre que la Byzance du Bas-Empire et du Moyen Âge n'a, de ce point de vue, pas volé sa réputation. L'intempérance interprétative caractérise au moins l'une des deux catégories d'interprètes que distingue un auteur du XIII ${ }^{\mathrm{e}}$ siècle, Sophonias, dans le préambule qu'il donne à sa paraphrase du De anima d'Aristote : à côtés des exégètes proprement dits (à ce groupe appartiennent les Alexandrins comme Ammonius, Simplicius, Philopon, mais aussi des érudits comme Alexandre d'Aphrodise), qui s'intéressent au texte lui-même et tentent d'éclairer la doctrine qu'ils transmettent, Sophonias fait état de pseudoparaphraseurs qui tentent d'interpréter les obscurités du discours des grands philosophes grecs (notamment Platon et Aristote) comme s'il s'agissait d'un discours divinatoire ou oraculaire. Les deux figures dominantes de ce courant, particulièrement représentatifs, selon $\mathrm{L}$. Brisson, de la réception de la philosophie grecque par le monde byzantin, que sont Psellos, auteur du xi ${ }^{\mathrm{e}}$ siècle, et son suiveur Pléthon, nous donnent une assez triste image de ce que peut être l'excès interprétatif d'une pratique herméneutique intempérante. Ils lisaient certes Platon et Aristote dans le texte, mais l'interprétation qui leur était naturelle leur venait des néo-platoniciens d'Athènes et d'Alexandrie qui cherchaient d'entrée de jeu, dans le cadre d'un enseignement encyclopédique rendant indissociables rhétorique et philosophie, un accord entre toutes les philosophies et toutes les théologies, entre la philosophie et la théologie, entre la théologie chrétienne et toutes les autres théologies. Psellos réalise la double synthèse entre les théologies païennes, grecques et barbares d'une part, et entre ces théologies païennes et la théologie chrétienne de l'autre, au prix d'une promotion douteuse de Platon en théologien, le Platon de la seconde partie du Parménide qui aurait transmis à des initiés une doctrine religieuse en accord avec toutes autres religions, $\mathrm{y}$ compris et avant tout avec le Christianisme. Pléthon reprendra en les accentuant à l'extrême les méthodes herméneutiques de Psellos, pour aboutir à des résultats opposés, comme la négation du Christianisme. L'excès interprétatif, sous la forme d'un usage complètement effréné de l'allégorie, était alors certes le prix à payer pour se faire l'héritier de la culture grecque à l'intérieur d'une religion qui régnait sans partage, et qu'animait une spiritualité monastique n'ayant que haine et mépris pour l'hellénisme qu'il confondait avec le paganisme : il fallait bien rendre acceptable à ses destinataires chrétiens la philosophie en la soumettant à une interprétation apologétique " tirée par les cheveux ».

Or, de ce point de vue (le point de vue des «cheveux tirés»), la Modernité est justement porteuse, plus qu'aucune autre époque, du risque d'intempérance interprétative. Il suffira de spécifier son byzantinisme pour surmonter l'objection de la dilution de sa spécificité dans un genre inadapté à sa réalité historique sui generis. Sans doute les temps ont-ils changé, et les mobiles apologético-religieux du byzantinisme 
proprement dit, disparu. Mais la générosité herméneutique de la Modernité ne cède en rien à celle de Byzance, en termes d'excès quantitatifs et qualitatifs. N'avons-nous pas, nous aussi, nos Psellos et nos Pléthon? Chacun nous accordera, espérons-le avec Umberto Eco, que "l'œuvre» est devenue un peu trop «ouverte», ou que, pour reprendre sa formule, « au cours des dernières décennies les droits des interprètes ont pris des proportions exagérées ", d'où les protestations sémiologiques contre la démesure des interprètes déconstructionnistes, habiles, il est vrai, tant à échafauder des compréhensions oiseuses et excessivement subtiles qu'à ébranler des compréhensions rigoureuses. U. Eco ne va pas chercher, comme G. Steiner, la pratique byzantine historique de l'interprétation pour modéliser les excès de l'école déconstructionniste, puisqu'il pousse la malice jusqu'à prétendre, aveu de caricature étant fait, que les théories de J. Derrida à propos de l'interprétation sont une excellente mise en théorie des pratiques du II $^{\mathrm{e}}$ siècle placées sous l'égide d'Hermès Trimégiste, l'Hermétisme de la Gnose. L'alchimie de Derrida ne vaut-elle pas la pharmacie de Platon? Il s'agit bien sûr d'une caricature, mais la caricature a ceci de bon que, n'étant pas un bon portrait de ce qui est, elle reste un bon portrait de ce qui pourrait être, si les hypothèses de départ les plus radicales orientant l'interprétation vers le lecteur et son soi-disant statut d'unique foyer du sens du texte étaient poussées à bout: la dégradation de l'herméneutique théorique et pratique en néo-hermétisme, en "gnosticisme textuel contemporain", comme dit U. Eco. La notion de byzantinisme apparaît alors dans sa valeur véritable, qui est plus programmatique et prospective que descriptive: avertir la Modernité de sa tendance à goûter un mauvais infini interprétatif, l'infini d'une libre interprétation qui, au lieu d'assumer sa liberté en avouant que le texte n'est pour elle qu'un prétexte à faire être ludiquement du sens, continue, non sans mauvaise foi peut-être, à prétendre que ses inventions s'enracinent malgré tout dans la mobilité signifiante du texte.

15 Cette précision étant faite, on peut envisager de laver la notion de byzantinisme du soupçon d'anti-intellectualisme que nous avons fait peser sur elle en début d'analyse. Si parler de byzantinisme, ce n'est pas nécessairement interdire à l'intelligence de s'exercer herméneutiquement, c'est parce que cela signifie bien plutôt lui demander de réguler son exercice. Mais il ne s'agit pas de lui faire accepter la nécessité de règles, car les règles n'ont jamais empêché l'excès interprétatif. Les pratiques les plus intempérantes de l'interprétation montrent au contraire que l'intempérance se joue surtout au niveau des règles elles-mêmes, c'est-à-dire que l'excès n'est pas un excès chaotique, mais est un excès organisé par une régulation elle-même excessive. Ce qui est frappant et instructif dans ses pratiques, qu'il s'agisse de celle de l'hermétisme gnostique, de l'allégorisme byzantin, ou du "gnosticisme textuel contemporain », c'est l'hyper-normativité de leurs productions. Par conséquent, une limitation de l'interprétation par régulation serait donc beaucoup plus avisée si elle se situait dans une exigence de règles pour les règles, c'est-à-dire du côté de l'examen de ces règles par ce qu'on pourrait appeler, à la manière kantienne, le tribunal de la raison.

Nous nous en tiendrons à deux illustrations, celles qu'on découvre en examinant la critique platonicienne des méthodes herméneutiques des sophistes. On se souvient qu'au début du Phèdre ${ }^{9}$, Socrate, décevant le jeune homme amateur d'allégories physicalistes des mythes, congédie (khairein - mots à mots : «Bien le bonjour !») les fables et renonce à les rationaliser, sous le prétexte étonnant parce qu'apparemment paresseux et futile, qu'il y faut, nous citons, «trop de génie, trop d'application laborieuse », et que "l'on y trouve pas du tout le bonheur». Si on entreprend de 
ramener chacun de ces êtres légendaires à la mesure de la vraisemblance en les remettant d'aplomb (éparnorthousthai), ajoute Socrate, on n'aura pas le temps de beaucoup flâner. Socrate poursuit, en donnant la vraie raison de la façon cavalière avec laquelle il envoie promener les mythes et leur interprétation allégorique : c'est qu'il trouve ridicule, ignorant qui il est, de se montrer curieux pour les choses étrangères, c'est-à-dire de délaisser le "Connais-toi » philosophique en pratiquant le « Connais-le » herméneutique. Quelle est la règle de règle qui commande en sourdine ces propos? C'est celle d'une précession de «l'herméneutique de soi » sur celle d'autrui. Générosité herméneutique bien ordonnée commence par soi-même, c'est-à-dire que l'examen de soi prime absolument l'examen des choses étrangères, lequel n'est tolérable (Socrate passe bien sa vie à examiner dialogiquement les pensées d'autrui) qu'à l'expresse condition, rappelée par Socrate à Critias dans le Charmide ${ }^{10}$, d'avoir pour but l'examen de soi. On peut dire que nous avons affaire ici à une régulation supérieure de l'interprétation, en ce sens qu'il ne s'agit pas d'énoncer une règle simplement herméneutique pour bien interpréter, mais une règle philosophique pour bien user de l'interprétation. Une herméneutique d'autrui indépendante du souci de soi est déjà, ipso facto, une interprétation qualifiable de byzantine, c'est-à-dire qui excède les limites d'une herméneutique philosophiquement réglée par l'impératif de réflexivité de l'intelligence. Il n'est donc pas soutenable de qualifier d'anti-intellectualisme l'exigence socratique d'avoir pour souci premier et dernier l'intelligence de soi par soi. Au contraire: c'est bien plutôt par intellectualisme philosophique que le Socrate platonicien envoie promener les mythes! C'est encore en suivant les leçons de Platon que l'on pourrait comprendre en quoi l'accusation de byzantinisme peut être lavée du soupçon de l'oubli du langage.

17 Une autre illustration de la nécessité de réguler philosophiquement les règles interprétatives se trouve dans le Protagoras, où ${ }^{11}$ un duel herméneutique semble opposer le philosophe et le sophiste, chacun redoublant de subtilité pour comprendre le poème de Simonide. Si Socrate accepte un moment le jeu proprement byzantin de Protagoras (c'est-à-dire excessivement subtil, comme l'atteste l'aspect douteux et extrêmement contorsionné du commentaire de Simonide par Socrate, comme n'ont pas manqué de le remarquer les critiques), c'est pour se livrer, immédiatement après, à une véritable palinodie, en mettant abruptement fin à tout conflit éristique des interprétations, par l'énoncé d'une règle fondamentale : donner congé aux poètes et ne recourir, dans le dialogue, qu'à nos voix propres, à nos propres ressources en matière de discours, et mettre à l'épreuve la vérité de ces discours, au lieu de faire porter l'examen sur ce que les poètes ont dit. Ce que Platon dénonce ici, dans le byzantinisme des sophistes, ce n'est pas autre chose que leur hétéronomie discursive, c'est-à-dire l'emprunt d'un discours étranger faute d'en avoir un propre et d'être capable, ou d'avoir le courage, de l'examiner. L'emprunt des poèmes est comparé, non sans mépris, à la location par des banqueteurs grossiers de danseuses et de joueuses de flûte, destinées à cacher l'indigence discursive des convives. Y a-t-il là oubli du langage, ou, tout au contraire, l'exigence d'une posture prise par le moi à l'égard du langage ? Platon demande une posture d'engagement et appropriation courageuse du langage, celle-la même que réclament les herméneutiques de Gadamer et de P. Ricœur ainsi que les analyses de G. Steiner. Là encore, ce n'est pas par oubli du langage que l'on s'autorisera à employer la notion de byzantinisme, mais par amour philosophique du langage, par «philologie» socratique. Cela étant dit, l'application de cette notion à 
l'exemple des sophistes grecs montre que nous n'avons pas achevé de spécifier le byzantinisme de la Modernité. Nous nous y emploierons pour conclure.

Conclusion

18 Pour saisir en quoi le byzantinisme de la Modernité est sui generis, et dépasse les byzantinismes théologiques ou sophistiques de l'histoire, il convient de diagnostiquer l'excès interprétatif moderne. Si la Modernité mérite le nom d'âge de l'interprétation, encore faut-il préciser de quelle interprétation on parle, en cherchant quelle origine on lui assigne. La perspective généalogique étant étrangère à U. Eco ainsi qu'à d'autres théoriciens de la portée démesurée prise par le concept d'interprétation que sont, entre autres, R. Shusterman pour les États-Unis, et J. Bouveresse pour la France ${ }^{12}$, nous préférons nous tourner vers le généalogiste philosophique par excellence qu'est Nietzsche. Cette référence est éminemment stratégique, en ce sens que, comme nous l'avons vu, le byzantinisme contemporain pourrait fort bien se réclamer du concepteur du « Nouvel infini » interprétatif, annoncé dans le § 374 du Gai Savoir. Or, un examen un peu plus attentif de la position de Nietzsche, en particulier du rapport précis qu'il établit justement entre interprétation et Modernité, suffirait à empêcher la récupération un peu rapide qu'on est tenté d'en faire. Ce que nous enseigne Nietzsche, c'est qu'annoncer un nouvel infini de l'interprétation n'est pas encourager le byzantinisme. Voyons, pour finir, comment il chemine sur la ligne de crête qui surplombe, d'un côté, la clôture dogmatique des signes, et de l'autre, le mauvais infini de l'ouverture byzantine.

On lit, dans La Volonté de Puissance ${ }^{13}$, sous le titre Pour une caractérisation de la Modernité, ceci : "- développement exagéré des formes intermédiaires». Nietzsche précise en énumérant ces formes: "La prééminence des marchands et des tiers, même dans le domaine intellectuel: le littérateur, le "représentant", l'historien (comme un amalgameur du passé et du présent), l'exotique et le cosmopolite, les intermédiaires entre les sciences naturelles et la philosophie, les semi-théologiens ». Nous ne sommes pas encore dans le contexte d'un excès herméneutique proprement dit: Nietzsche se contente ici d'élucider la Modernité comme un excès dans la médiation en général, quel qu'en soit le type: la médiation économique (le marchand s'entremet entre le producteur et le consommateur); la médiation temporelle (l'historien qui cherche à vaincre la distance entre passé et présent); la médiation politique (le politicien ou le syndicaliste qui prétendent représenter le peuple, un parti ou un groupe d'intérêt dans les assemblées); la médiation esthétique (le poète veut faire croire qu'il n'est qu'un porte-parole); la médiation religieuse (le prêtre qui, selon Nietzsche, cherche à faire accroire aux foules que lui seul est l'intermédiaire entre celles-ci et Dieu). Pour Nietzsche, la Modernité est donc un âge de l'interprétation parce qu'elle est la véritable ère de la médiation, des médiateurs et des médias. Or, en tant que penseur, il fait un sort à l'espèce intellectuelle de la médiation. C'est dans cette espèce, justement, que l'on va trouver la place de l'excès interprétatif.

Que l'interprétation appartienne au genre de la médiation, ce n'est pas difficile à comprendre, à condition que l'on se souvienne qu'elle n'est pas binairement structurée, comme on la décrit trop souvent. Interpréter n'est pas le rapport simplement dialogique entre le seul interprète et le phénomène, l'œuvre, l'auteur, le discours, la loi, la partition, les symptômes ou les textes sacrés: toute inter-prétation semble bien plutôt interprétation de quelque chose par quelqu'un pour quelqu'un, c'est-à-dire qu'elle est ternairement constituée. Hermès est le dieu messager entre les dieux et les hommes ; 
l'exégète est un inter-cesseur entre les fidèles et le dieu dont il éclaire les dires ; c'est par l'entremise du devin que les signes des dieux sont compris par les hommes à qui ils sont envoyés ; le traducteur-interprète sert d'intermédiaire entre deux personnes qui ne sont pas isoglosses ; se faire l'interprète de quelqu'un auprès de quelqu'un, n'est-ce pas lui servir de truchement? Le jurisconsulte se situe entre la législation et les citoyens auxquels celle-ci veut s'appliquer ; le psychanalyste, quant à lui, ne sert-il pas de médiateur à son patient en manque d'unité psychique? Le commentateur, lecteur professionnel, s'interpose entre le texte et ses lecteurs, comme le critique d'art, goûteur professionnel, entre l'artiste et son public. Qu'on examine toutes les espèces, aussi bien pratiques que théoriques, de l'interprète, le point commun reste celui d'une fonction de médiation verbale entre deux instances primitivement séparés par leurs langages étrangers et que l'interprète cherche à réunir dans la communauté d'un langage nouveau surmontant leur scission originelle. On pourrait donc diagnostiquer l'excès interprétatif moderne comme étant l'effet peut-être panique d'une prise de conscience grandissante de la scission qui nous sépare de nous-mêmes. La finitude de la conscience serait, dans cette hypothèse, responsable du byzantinisme moderne, effort intempérant et vain pour surmonter cette finitude, et en même temps symptôme éminent de la prise de conscience de celle-ci.

Mais Nietzsche ne se livrera pas à une telle analyse, et nous ne suivrons pas plus loin cette piste qui mériterait des développements. C'est le thème de la décadence culturelle, manifestée par une irritabilité et une réactivité maladives, qui sera par lui chargée d'expliquer la tendance moderne à l'excès interprétatif. Le médiateur est médiateur en tant qu'il est un réactif. L'interprète intempérant, espèce de médiateur intellectuel, ne déroge pas à cette condition décadente. Dans le $\S 44$, sous le titre $L a$ Modernité envisagée sous le double symbole de la nutrition et de la digestion, nous lisons : « La sensibilité est infiniment plus irritable [...] l'abondance des impressions disparates est plus grande que jamais : - le cosmopolitisme des langues, des littératures, des journaux, des formes, des goûts différents, même des paysages. L'allure de cette affluence est un prestissimo; les impressions s'effacent; on se défend instinctivement d'absorber quelque chose, de s'en laisser impressionner profondément, de "digérer" quelque chose ; il en résulte l'affaiblissement de la faculté de digestion ». Nietzsche a bien en vue les interprètes, c'est-à-dire les médiateurs culturels, comme il le précise un peu plus loin (nos derniers mots seront pour lui laisser la parole): "Il se produit une sorte d'assimilation à cette accablement d'impressions; l'homme désapprend d'agir; il ne réagit plus qu'à des impressions du dehors. Il dépense ses forces, soit dans l'assimilation, soit dans la défense, soit dans la réplique. Profond affaiblissement de la spontanéité: l'historien, le critique, l'analyste, l'interprète, l'observateur, le collectionneur, le lecteur - ils sont tous des talents réactifs -, ils font tous partie de la science !».

\section{NOTES}

1.. G. STEINER, Le Sens du sens, Paris, Éd. Vrin, 1988, p. 47. 
2.. Puisqu'elle s'étend sur plus de mille ans! Et qu'elle a permis, pour l'essentiel, la sauvegarde de la culture et de la philosophie antiques dont elle s'est faite malgré tout l'héritière. Nous renvoyons, pour en prendre une mesure exacte dans le domaine de la philosophie, aux très bonnes pages que lui consacre L. BRISSON dans l'ouvrage collectif paru en 1997, Philosophie grecque, huitième partie, intitulée « Le monde byzantin et la philosophie grecque », Paris, PUF, p. 745-779.

3.. Cf. les deux ouvrages de Umberto ECO, Les Limites de l'interprétation, paru en 1992, Paris, Grasset, trad. M. Bouzahert, et Interprétation et surinterprétation, Paris, PUF, 1996, trad. J.-P. Cometti.

4.. F. FÉDIER, Interprétations, Paris, PUF, 1985, p. 12.

5.. DU BOS, Journal, 29.09.1917.

6.. R. BARTHES, Critique et vérité, Paris, Seuil, 1966, p. 45.

7.. J. PÉPIN, «L'herméneutique ancienne, les mots et les idées », in Poétique, Revue de théorie et d'analyse littéraire, Paris, Seuil, 1975, p. 291-300.

8.. U. ECO, Interprétation et surinterprétation, op. cit., p. 22.

9.. Euvres complètes, trad. L. Robin, Paris, Éd. Les Belles Lettres, 1944, tome IV, $3^{\mathrm{e}}$ partie, 229c-230a.

10.. Trad. A. Croiset, Paris, Éd. Les Belles Lettres, 1921, 166d.

11.. Euvres complètes, trad. L. Bodin, Paris, Éd. Les Belles Lettres, 1967, tome III, $1^{\text {ère }}$ partie, de 339 à 347.

12.. Nous renvoyons à leurs travaux tout à faits adaptés à la discussion, qui montrent les risques d'une extension trop importante du concept d'interprétation dont est grosse l'herméneutique gadamérienne, respectivement : Sur l'interprétation, Combas, Éd. de l'Éclat, coll. «Tiré à part », 1994, trad. J. Cometti ; et Herméneutique et linguistique, Combas, Éd. de l'Éclat, coll. « Tiré à part », 1991.

13.. Trad. H. Albert, Paris, 1991, § 48.

\section{RÉSUMÉS}

Plus qu'un événement ou un objet, l'interprétation est un philosophème, prégnant au $\mathrm{xx}^{\mathrm{e}}$ siècle, qui ne se borne pas à désigner un concept mais toute une méthodologie. Sans doute la notion et la pratique de l'interprétation ne sont-elles pas nouvelles. Mais il semble que le propre de la Modernité a été de leur assurer une fortune sans précédent. Sécularisée par le XIX ${ }^{e}$ siècle qui l'a redécouverte à l'occasion du problème des études historiques, l'interprétation s'est étendue, en théorie comme en pratique, et en particulier par le relais de la psychanalyse, à l'ensemble de la culture, au risque de faire d'elle une culture « byzantine». 